\title{
Clinical significance of quantitative HER2 gene amplification as related to its predictive value in breast cancer patients in neoadjuvant setting
}

This article was published in the following Dove Press journal:

OncoTargets and Therapy

\section{Ziping $\mathrm{Wu}^{1, *}$ \\ Shuguang $\mathrm{Xu}^{1, *}$ \\ Liheng Zhou' \\ Wenjin Yin ${ }^{2}$ \\ Yanpin Lin' \\ Yueyao $\mathrm{Du}^{\prime}$ \\ Yaohui Wang' \\ Yiwei Jiang' \\ Kai Yin' \\ Jie Zhang' \\ Jinsong $\mathrm{Lu}^{\prime}$}

'Department of Breast Surgery, Renji Hospital, School of Medicine, Shanghai Jiao Tong University, Shanghai, People's Republic of China; ${ }^{2}$ Cancer Institute, Fudan University Shanghai Cancer Center, Fudan University, Shanghai, People's Republic of China

*These authors contributed equally to this work
Correspondence: Jinsong Lu

Department of Breast Surgery, Renji Hospital, School of Medicine, Shanghai Jiao Tong University, No 160 Pujian Road, Shanghai 200127 ,

People's Republic of China

Email lujjss@I63.com
Background: The aims of this study were to determine whether the quantitative HER2 gene amplification level is related to the key clinicopathological features that represent the aggressiveness of breast cancer (BC) and to determine whether the quantitative HER2 gene amplification level could predict the treatment response in the subset of HER2-positive patients who received neoadjuvant targeted therapy.

Materials and methods: Patients treated with weekly cisplatin- and paclitaxel-based neoadjuvant chemotherapy, who had undergone both immunohistochemistry and the fluorescence in situ hybridization test for HER2, were included in the study ( $\mathrm{n}=103)$. For HER2-positive patients, defined as immunohistochemistry score $3+$ or fluorescence in situ hybridization ratio $\geq 2.0$, trastuzumab was recommended with neoadjuvant chemotherapy $(n=45)$. Pathological complete response was defined as complete pathological remission of tumor cells both in breast and axillary lymph nodes postoperation.

Results: In all patients enrolled in the study, a higher HER2 amplification level was significantly correlated with larger tumor size and the absence of ER and PR expression. In HER2-positive patients treated with neoadjuvant trastuzumab concurrent with chemotherapy, both univariate and multivariate logistic regression showed that a higher HER2/CEP17 ratio and HER2 gene copy number were associated with a higher pathological complete response rate. When calculated by receiver operating characteristics analysis, an optimal cutoff of 4.5 for the HER2/CEP17 ratio was expected to distinguish the most sensitive candidate for treatment with a combination of trastuzumab and neoadjuvant chemotherapy.

Conclusion: A higher HER2 amplification level was correlated with larger tumor size and reduced ER and PR expression, which may indicate more aggressive tumor behavior. For HER2-positive patients, the HER2/CEP17 ratio and HER2 gene copy number may be good predictive factors for concurrent neoadjuvant trastuzumab and chemotherapy.

Keywords: breast cancer, neoadjuvant, quantitative HER2 gene amplification, pathological complete response, predictive

\section{Introduction}

The HER 2 gene encodes a $185 \mathrm{kDa}$ monomeric protein, which is a receptor tyrosine kinase that belongs to the human epidermal growth factor receptor family. ${ }^{1}$ Approximately $25 \%$ of breast cancer (BC) patients present with HER2 gene amplification, ${ }^{1}$ which is an independent prognostic factor of poor outcome. Anti-HER2 therapy is now a standard treatment for HER2-positive patients both in adjuvant and metastatic settings. ${ }^{2}$ When HER2-positive patients are treated with adjuvant trastuzumab therapy, the 10 -year survival rate could be increased from $75.2 \%$ to $84 \%$. 
Still, approximately $10 \%$ of these patients will inevitably develop recurrent or metastatic disease despite anti-HER2 treatment, reflecting the heterogeneous tumor biology of HER2-positive BC. ${ }^{3}$

In addition to the well-acknowledged fact that HER2 expression status represents a poor prognosis and good response to trastuzumab, effective methods are still required to distinguish the tumor biology of HER2-positive BC. As a routinely conducted test, fluorescence in situ hybridization (FISH) provides a linear measurement for the HER2 gene amplification level, which seems to be a promising figure in reflecting the nature of HER2-positive BC. Several studies were carried out referring to this issue in adjuvant, neoadjuvant, and metastatic settings. In adjuvant settings, a large sample study by Xuan et $\mathrm{al}^{4}$ observed an increased disease-free survival in trastuzumab-treated patients with a lower HER2 amplification level. In addition, a study from Borley et $\mathrm{al}^{5}$ observed an increased progression-free survival and overall survival in patients with lower HER2 gene copy number. However, research that was correlated to the HERA trial showed no obvious influence of the HER2 amplification degree on patient prognosis. ${ }^{6}$ The latest meta-analysis from $\mathrm{Xu}$ et $\mathrm{al}^{7}$ denied any correlations between this figure and survival.

In late-stage $\mathrm{BC}$, a study from Fuchs et $\mathrm{al}^{8}$ showed both higher HER2 gene copy number (GCN) and that HER2/ $C E P 17$ ratio is associated with shorter time to first metastasis in patients treated with trastuzumab and chemotherapy. Similarly another study also observed a shorter time to progression in patients with high $H E R 2 / C E P 17$ ratio. ${ }^{9}$ However, there was another study that observed an opposite trend: patients with high-level HER2/CEP17 ratio showed a longer progression-free survival. ${ }^{10}$

In regard to neoadjuvant settings, results from different institutions were even more controversial. ${ }^{1-13}$ It is still unclear whether HER2 amplification level is correlated with patients' response to concurrent neoadjuvant therapy.

Disparity in the results may derive from the heterogeneity of patients and treatment in each study. Thus, we started to investigate this issue in the population of our prospective trials, in which patients received weekly paclitaxel in combination with cisplatin for neoadjuvant chemotherapy concurrent with trastuzumab in cases where the tumor was HER2-positive. In this study, we hypothesize that a higher HER2/CEP17 ratio or HER $2 \mathrm{GCN}$ is associated with more aggressive tumor biology and better pathological complete response (pCR) outcome in this highly uniform subset of patients.

\section{Materials and methods Patients and study design}

$\mathrm{BC}$ patients from two paclitaxel- and cisplatin-based neoadjuvant clinical trials were included. The two trials were separately registered in ClinicalTrials.gov as SHPD001 (NCT02199418) and SHPD002 (NCT02221999). SHPD001 was reviewed and approved by two independent ethical committees and institutional review boards of RenJi Hospital, Shanghai Jiao Tong University and Fudan University Shanghai Cancer Center. SHPD002 was reviewed and approved by the independent ethical committee and institutional review board of RenJi Hospital, Shanghai Jiao Tong University. All patients provided written informed consent.

Women aged $\geq 18$ years old with histologically confirmed locally advanced invasive BC were included. For all patients, paclitaxel $80 \mathrm{mg} / \mathrm{m}^{2}$ was given weekly starting on day 1 for 16 weeks; cisplatin $25 \mathrm{mg} / \mathrm{m}^{2}$ was given weekly on day 1,8 , and 15 every 28 days for 4 cycles. For HER2-positive patients in SHPD001, trastuzumab was recommended concurrently. All HER2-positive patients in SHPD002 received concurrent trastuzumab. Trastuzumab was given at a loading dose of $4 \mathrm{mg} / \mathrm{kg}$, followed by a maintenance dose of $2 \mathrm{mg} / \mathrm{kg}$, on day 1 for 16 weeks. For hormone receptor-positive patients in SHPD002, endocrine therapy of aromatase inhibitor or gonadotropinreleasing hormone agonist was randomized together with chemotherapy according to the patient's menstrual status. Planned surgery was given sequentially after neoadjuvant chemotherapy. All procedures performed in studies involving human participants were in accordance with the ethical standards of the institutional and/or national research committee and with the 1964 Helsinki declaration and its later amendments or comparable ethical standards.

The study's primary outcome with regard to the two groups SHPD001 and SHPD002 was pCR, which was defined as the absence of tumor in the breast and axillary lymph nodes samples taken at the time of surgery.

Up until December 2016, 103 postoperative patients who went through the FISH test and 45 HER2-positive trastuzumabtreated patients were available for the analysis.

\section{$\mathrm{IHC}$ and $\mathrm{FISH}$}

ER, PR, and ki67 levels were assessed by immunohistochemistry (IHC) in paraffin-embedded tumor samples from biopsy. All patients enrolled in the clinical trial were tested by IHC to determine their HER2 status. Patients who tested 1+ to 3+ by IHC and some of the IHC 0 patients $(n=103)$ were examined 
by FISH. FISH was carried out on paraffinized tissue sections using the HER2 DNA Dual Probe Kit (Linked-Biotech Pathology, Guangzhou, People's Republic of China). HER2 positivity is defined according to American Society of Clinical Oncology (ASCO)/College of American Pathologists (CAP) recommendations from $2013 .{ }^{14}$ In detail, 60 cells were routinely counted for FISH testing. Each cell was counted for HER2 signal (red) and CEP17 signal (green). Then, an average $H E R 2$ signal per cell and an average $C E P 17$ signal per cell were used to obtain the final FISH ratio.

Both IHC and FISH assessment were conducted and confirmed by two qualified pathologists from the Department of Pathology, Shanghai Renji Hospital or Fudan University Shanghai Cancer Center.

\section{Statistical methods}

Spearman's correlation test was used to determine the correlations between the HER2 GCN, HER2/CEP17 ratio, and other clinicopathological characteristics in patients who underwent FISH ( $\mathrm{n}=103)$. In patients treated with concurrent trastuzumab $(\mathrm{n}=45)$, correlations between HER2 $\mathrm{GCN}$, the HER2/CEP17 ratio, and pathological response to neoadjuvant therapy were calculated by both univariate and multivariate logistic regression analysis. Receiver operating characteristic (ROC) analysis was performed to see if HER2 GCN and HER2/CEP17 could independently predict patients' response to neoadjuvant therapy. Sensitivity and specificity were calculated to determine the optimal cutoff points. All statistical analyses were carried out using STATA Statistics SE 14 (Stata Corp LP, College Station, TX, USA). The results were considered significant when $p<0.05$.

\section{Results}

\section{Correlations of HER2 gene amplification level and other clinicopathological characteristics}

The baseline characteristics of all 103 patients who were available for FISH results to be obtained are shown in Table 1. Among these patients, HER2 GCN ranged from 1.45 to 65 and HER2/CEP17 ratios ranged from 1.09 to 50 . No polysomy 17 cases were reported. HER2 GCN was inversely related to ER expression level $(p<0.001)$ and PR expression level $(p<0.001)$ and positively related to ki67 level $(p=0.042)$; the HER 2/CEP17 ratio was also inversely related with ER expression level $(p<0.001)$ and PR expression level $(p<0.001)$ and positively related with tumor size $(p=0.004)$. No significant correlations were found between age and the HER 2 amplification level in either the ratio or GCN group (Table 2).
Table I Baseline clinical characteristics of all patients

\begin{tabular}{lll}
\hline Characteristics & Number (n) & Percentage (\%) \\
\hline Age (years) & & \\
$\quad \geq 50$ & 59 & 57 \\
$\quad<50$ & 44 & 43 \\
Tumor size & & \\
$\quad>5 \mathrm{~cm}$ & 58 & 56 \\
$\quad \leq 5 \mathrm{~cm}$ & 44 & 44 \\
ER status & & \\
ER + & 78 & 76 \\
ER - & 25 & 24 \\
PR status & & \\
PR + & 85 & 83 \\
PR - & 18 & 17 \\
ki67 status & \\
$>30 \%$ & & \\
$\quad \leq 30 \%$ & 59 & 57 \\
Menopause status & 44 & 43 \\
Pre & & \\
Post & 51 & 50 \\
\hline
\end{tabular}

Note: ${ }^{2}$ Most of the patients had a ki67 level greater than $14 \%$, and thus $30 \%$ was used to separate the groups.

\section{Baseline clinical characteristics of trastuzumab-treated patients and their response to chemotherapy concurrent with target therapy}

Among the 45 patients who were HER2 positive and treated with trastuzumab, 22 patients reached pCR after surgery; the pCR rate was $48.9 \%$. The baseline clinical characteristics of these 45 patients are summarized in Table 3 . The detailed relationship between the HER2/CEP17 ratio, HER2 GCN, and neoadjuvant outcome for each patient is displayed in Figures 1 and 2. An obvious increase in pCR outcome was observed when the HER2/CEP17 ratio or HER2 GCN was elevated.

\section{Correlations of quantitative HER2 gene amplification and $p C R$ outcome}

In univariate analysis, both a higher HER2/CEP17 ratio (odds ratio $[\mathrm{OR}]=1.827, p=0.008 ; 95 \%$ confidence interval $[\mathrm{CI}]$ :

Table 2 Correlations between HER2 amplification level and clinicopathological characteristics of all patients

\begin{tabular}{lllll}
\hline Characteristics & $\mathbf{N}^{\mathrm{a}}$ & $\begin{array}{l}\text { p-value } \\
\text { (HER2 GCN) }^{\mathrm{b}}\end{array}$ & $\mathbf{N}$ & $\begin{array}{l}\text { p-value } \\
\text { (HER2/CEP I7 } \\
\text { ratio) }\end{array}$ \\
\hline Age & 101 & 0.051 & 103 & 0.465 \\
Tumor size & 100 & 0.194 & 102 & $\mathbf{0 . 0 0 4}$ \\
ER status & 101 & $\mathbf{0 . 0 0 0}$ & 103 & $\mathbf{0 . 0 0 0}$ \\
PR status & 101 & $\mathbf{0 . 0 0 0}$ & 103 & $\mathbf{0 . 0 0 0}$ \\
Menopausal status & 101 & 0.057 & 103 & 0.372 \\
ki67 & 101 & $\mathbf{0 . 0 4 2}$ & 103 & 0.154 \\
\hline
\end{tabular}

Notes: a 101 patients had values for HER2 GCN; 'Bold facing indicates statistical significance.

Abbreviation: GCN, gene copy number. 
Table 3 Baseline clinical characteristics of HER2-positive trastuzumab-treated patients

\begin{tabular}{lll}
\hline Characteristics & Number (n) & Percentage (\%) \\
\hline Age (years) & & \\
$\quad \geq 50$ & 26 & 58 \\
$\quad<50$ & 19 & 42 \\
Tumor size & & \\
$\quad>5 \mathrm{~cm}$ & 30 & 67 \\
$\quad \leq 5 \mathrm{~cm}$ & 15 & 33 \\
ER status & & \\
ER + & 30 & 67 \\
ER - & 15 & 33 \\
PR status & & \\
PR + & 33 & 73 \\
PR - & 12 & 7 \\
ki67 status & & \\
$>30 \%$ & 30 & 67 \\
$\quad \leq 30 \%$ & 15 & 33 \\
Menopause status & & \\
Pre & 21 & 57 \\
Post & 24 & 53 \\
\hline
\end{tabular}

1.176-2.851) and HER2 GCN amplification level $(\mathrm{OR}=1.139$, $p=0.029 ; 95 \%$ CI: $1.013-1.230$ ) were associated with a better $\mathrm{pCR}$ rate, while ER expression was associated with a poorer pCR rate $(\mathrm{OR}=0.211, p=0.025 ; 95 \%$ CI: $0.053-0.824)$.

Similarly, in multivariate analysis, a higher HER2/CEP17 ratio $(\mathrm{OR}=2.110, p=0.005 ; 95 \% \mathrm{CI}: 1.266-3.861)$ was also associated with a better $\mathrm{pCR}$ rate, while ER expression was associated with a poorer $\mathrm{pCR}$ rate $(\mathrm{OR}=0.101, p=0.038 ; 95 \%$ CI: 0.012-0.884). HER2 GCN was also positively related to $\mathrm{pCR}(\mathrm{OR}=1.147, p=0.050 ; 95 \% \mathrm{CI}: 1.000-1.317)$ when adjusted. No significant correlations between other potential factors including patient age, PR status, and tumor size were observed (Tables 4-6).

\section{Optimal predictive cutoff points decided by ROC curve}

The optimal cutoff point determined by ROC analysis was 4.5 for the HER2/CEP17 ratio and 14 for HER2 GCN, which were expected to distinguish the most sensitive and specific candidates for cisplatin- and paclitaxel-based weekly neoadjuvant chemotherapy combined with trastuzumab. The area under the curve value of the HER2/CEP17 ratio was 0.786 compared with 0.656 of HER2 GCN (Figure 3).

\section{Discussion}

HER2 gene amplification was associated with poor survival outcome in $\mathrm{BC}$ patients. It is also an indication of trastuzumab treatment in HER2-positive BC patients. ${ }^{15}$ However, the dosage effect of the HER2 gene amplification level on $\mathrm{BC}$ patients is not quite clear. Our study demonstrated that both HER2 GCN and HER2/CEP17 ratios are correlated with unfavorable prognostic factors. As for the predictive value, we demonstrated that both the HER2/CEP17 ratio and HER GCN were associated with better pCR outcome in trastuzumab-treated patients. This was the first time, at least to our knowledge, that correlations between tumor biology features, neoadjuvant treatment sensitivity, and both the HER2/CEP17 ratio and HER2 GCN were demonstrated in data from prospective clinical trials in the People's Republic of China.

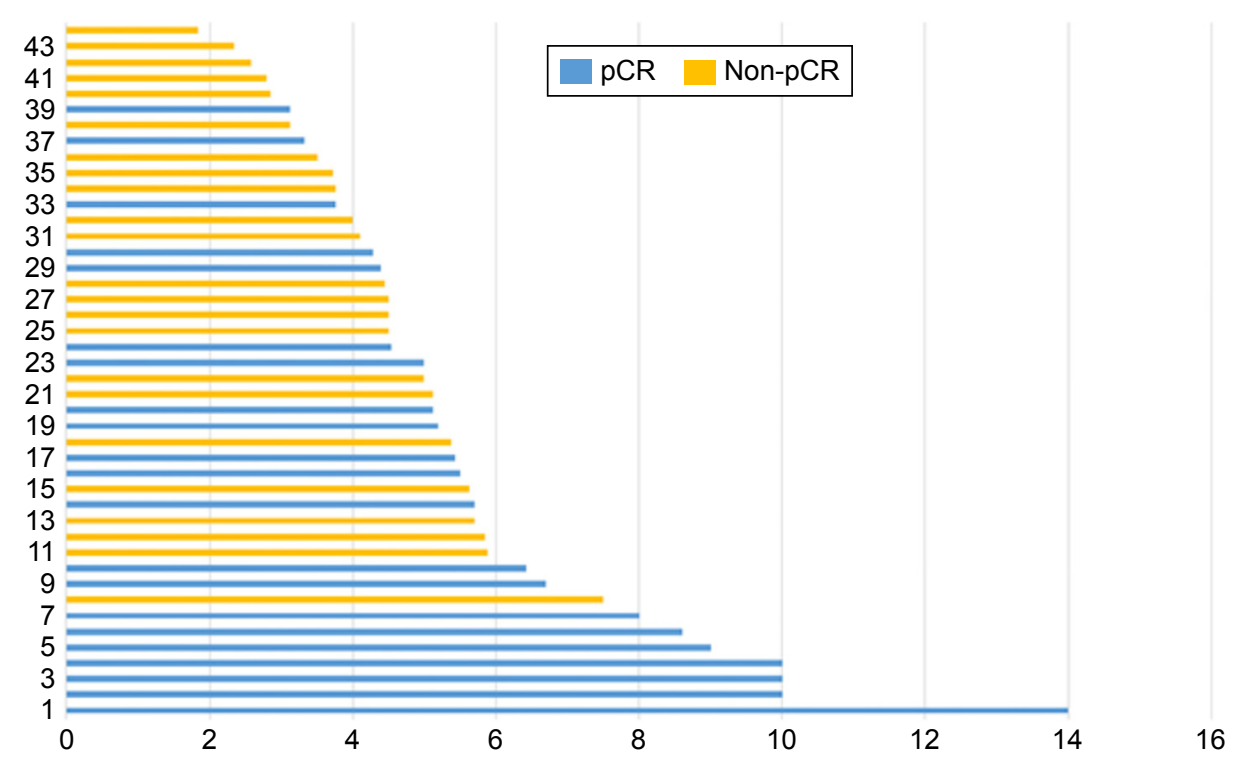

Figure I HER2/CEPI7 ratio and neoadjuvant outcome of 44 patients treated with trastuzumab.

Notes: Every column represents an individual patient with an HER2/CEPI7 ratio value (x-axis). The blue column represents pCR outcome, yellow column represents nonPCR outcome. An increasing tendency for PCR outcome was observed when the HER2/CEPI7 ratio was elevated. One patient was not shown in the figure because the HER2/CEPI7 value was too big (50).

Abbreviation: $\mathrm{PCR}$, pathological complete response. 


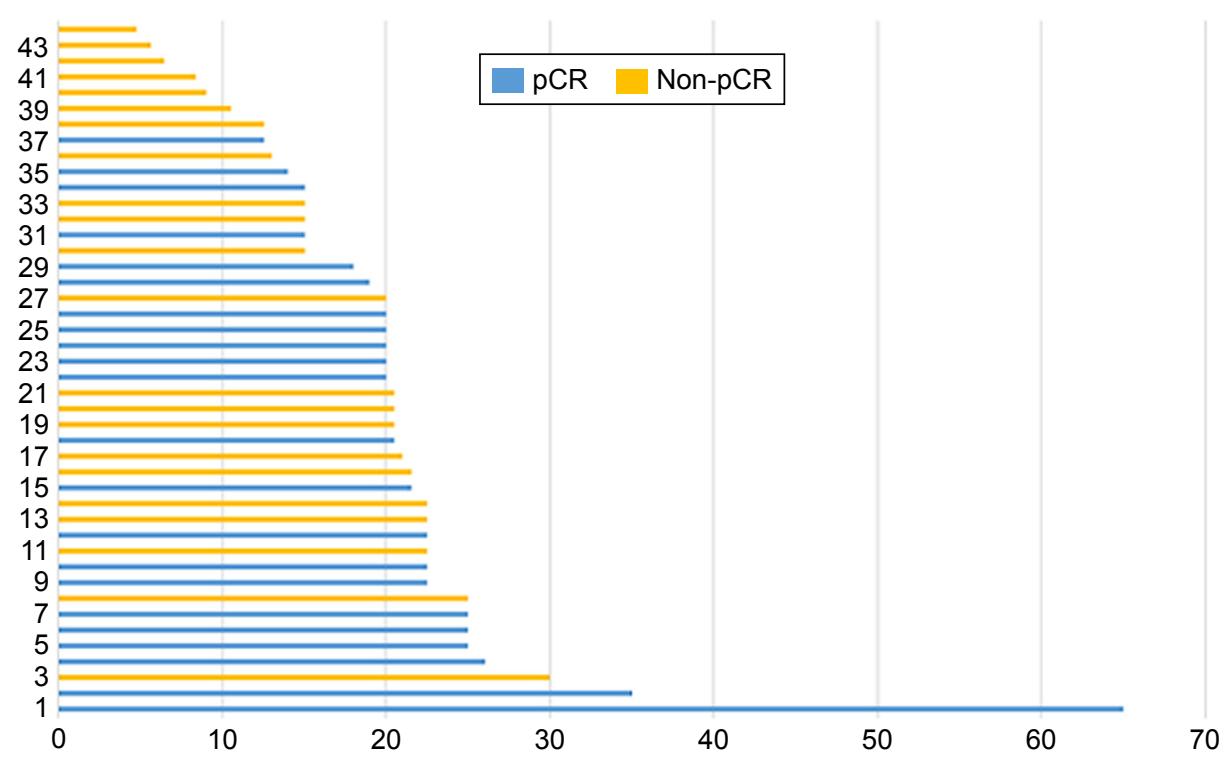

Figure 2 HER2 GCN and neoadjuvant outcome of 44 patients treated with trastuzumab.

Notes: Every column represents an individual patient with an HER2 GCN value (x-axis). The blue column represents pCR outcome, yellow column represents non-pCR outcome. An increasing tendency for PCR outcome was observed when HER2 GCN was elevated.

Abbreviations: GCN, gene copy number; $\mathrm{PCR}$, pathological complete response.

We did FISH tests in patients with an IHC score equal to or greater than 0 at biopsy. This provided a more diverse population compared to studies conducted in patients who were mostly IHC $3+$. In this set of patients, a higher HER2 amplification level seemed to be accompanied by unfavorable clinicopathological factors. For instance, ER and PR status were inversely related to both HER $2 \mathrm{GCN}$ and HER2/CEP17 ratios. This result was supported by the work from Arnould et al. ${ }^{11}$ In that research, high amplification of $H E R 2$ was correlated with hormone receptor-negative tumors.

With regard to predictive value, existing data from clinical trials and observations have already shown that tumors with higher proliferation and invasion potential were more sensitive to chemotherapy. For instance, triple-negative BC often exhibits an increased $\mathrm{pCR}$ rate but a decreased overall

Table 4 Univariate analyses for predictive factors of HER2positive trastuzumab-treated patients

\begin{tabular}{llllll}
\hline Characteristics & \multicolumn{6}{l}{ Univariate analysis } \\
\cline { 2 - 6 } & $\mathbf{N}$ & OR & $\mathbf{9 5 \%} \mathbf{C l}$ & p-value $^{\text {b }}$ \\
\hline HER2 GCN & $44^{\mathrm{a}}$ & $\mathrm{I} .139$ & $\mathrm{I} .013$ & $\mathrm{I} .230$ & $\mathbf{0 . 0 2 9}$ \\
HER2/CEPI 7 ratio & 45 & 1.827 & $\mathrm{I} .170$ & $2.85 \mathrm{I}$ & $\mathbf{0 . 0 0 8}$ \\
Age (years) $\geq 50$ vs $<50$ & 45 & 2.338 & 0.694 & 7.872 & 0.170 \\
ER positive vs negative & 45 & $0.21 \mathrm{I}$ & 0.053 & 0.824 & $\mathbf{0 . 0 2 5}$ \\
PR positive vs negative & 45 & 0.368 & 0.092 & $\mathrm{I} .47 \mathrm{I}$ & 0.158 \\
Tumor size (cm) $>5$ vs $\leq 5$ & 45 & 0.680 & 0.219 & 2.108 & 0.504 \\
Menopause status pre vs post & 45 & 1.576 & 0.484 & 5.126 & 0.450 \\
ki67 & 45 & 1.028 & 0.994 & 1.063 & 0.105 \\
\hline
\end{tabular}

Notes: ${ }^{a} 44$ patients had values for HER2 GCN; 'Bold facing indicates statistical significance.

Abbreviations: $\mathrm{OR}$, odds ratio; $\mathrm{Cl}$, confidence interval; $\mathrm{GCN}$, gene copy number. survival compared with other BC subtypes. ${ }^{16,17}$ Similar to triple-negative $\mathrm{BC}$, a study from $\mathrm{Yu}$ et $\mathrm{al}^{12}$ demonstrated that HER2-positive BC was sensitive to neoadjuvant chemotherapy. ${ }^{12}$ All the patients in that study were HER2 positive and received neoadjuvant chemotherapy without targeted therapy. Yu et al observed a better $\mathrm{pCR}$ rate in patients with both higher HER2/CEP17 ratio and HER2 GCN.

Yu et al's study might indicate the sensitivity of patients with higher HER2 amplification level to chemotherapy. Our study demonstrated, as well, that these patients are more sensitive to targeted therapy.

Our results are partially supported by the studies from Arnould et $\mathrm{al}^{11}$ and Guiu et $\mathrm{al},{ }^{15}$ where patients received concurrent trastuzumab and neoadjuvant chemotherapy of different regimens. Those patients were originally tested IHC $3+$ or $2+$ for HER 2 status. FISH assays were retrospectively conducted using the ASCO/CAP guideline version 2007. ${ }^{23}$

Table 5 Multivariate analyses for predictive factors of $p C R$ using HER2/CEPI 7 ratio

\begin{tabular}{llllll}
\hline Characteristics & \multicolumn{5}{l}{ Multivariate analysis } \\
\cline { 2 - 6 } & $\mathbf{N}$ & OR & $\mathbf{9 5 \%} \mathbf{C l}$ & p-value $^{\mathbf{a}}$ \\
\hline HER2/CEPI7 ratio & 45 & 2.210 & 1.266 & $3.86 \mathrm{I}$ & $\mathbf{0 . 0 0 5}$ \\
Age (years) $\geq 50$ vs $<50$ & 45 & 0.962 & 0.167 & 5.560 & 0.966 \\
ER positive vs negative & 45 & 0.078 & 0.009 & 0.673 & $\mathbf{0 . 0 2 0}$ \\
PR positive vs negative & 45 & 1.192 & 0.187 & 7.585 & 0.852 \\
Tumor size (cm) $>5$ vs $\leq 5$ & 45 & 0.719 & 0.480 & 1.077 & 0.110 \\
\hline
\end{tabular}

Note: ${ }^{a}$ Bold facing indicates statistical significance.

Abbreviations: $\mathrm{PCR}$, pathological complete response; OR, odds ratio; $\mathrm{Cl}$, confidence interval. 
Table 6 Multivariate analysis for predictive factors of $p C R$ using HER2 GCN

\begin{tabular}{llllll}
\hline Characteristics & \multicolumn{5}{l}{ Multivariate analysis } \\
\cline { 2 - 6 } & $\mathbf{N}^{\mathrm{a}}$ & OR & $\mathbf{9 5 \%} \mathbf{~ C l}$ & p-value $^{\mathrm{b}}$ \\
\hline HER2 GCN & 44 & 1.147 & 1.000 & 1.317 & $\mathbf{0 . 0 5 0}$ \\
Age (years) $\geq 50$ vs $<50$ & 44 & 0.609 & 0.117 & 3.176 & 0.556 \\
ER positive vs negative & 44 & 0.190 & 0.343 & 1.046 & 0.056 \\
PR positive vs negative & 44 & 0.560 & 0.125 & 2.723 & 0.473 \\
Tumor size $(\mathrm{cm})>5$ vs $\leq 5$ & 44 & $0.88 \mathrm{I}$ & 0.654 & 1.186 & 0.404 \\
\hline
\end{tabular}

Notes: ${ }^{4} 44$ patients had values for HER2 GCN; ${ }^{b}$ Bold facing indicates statistical significance.

Abbreviations: $\mathrm{pCR}$, pathological complete response; GCN, gene copy number; $\mathrm{OR}$, odds ratio; $\mathrm{Cl}$, confidence interval.

Only HER2 GCN was analyzed. When HER $2 \mathrm{GCN}=10$ was used as the cutoff, the authors observed a better $\mathrm{pCR}$ rate in the cohort with high GCN. Our work is also supported by the work from Kogawa et al. Patients who received concurrent trastuzumab and neoadjuvant chemotherapy in that study showed a positive correlations between HER2/CEP17 ratio and pCR. ${ }^{18}$

On the other hand, the study from Buzdar et $a{ }^{13}$ showed no correlation between HER2 amplification level and pCR outcome. However, only a brief conclusion was mentioned in the study. A detailed statistical method was not described.

Different from the neoadjuvant setting, results in the adjuvant settings are quite inconsistent. Briefly, we summarize some relevant studies in Table 7. In adjuvant settings, studies from Xuan et $\mathrm{al}^{4}$ and Borley et $\mathrm{al}^{5}$ found a positive correlation between HER2 GCN and survival, but studies from Perez et $\mathrm{al}^{3}$ and Dowsett et $\mathrm{al}^{6}$ found no correlation. The difference between settings might derive from the schedule of trastuzumab. In neoadjuvant settings, trastuzumab is often used concurrently with chemotherapy. But in the adjuvant setting, trastuzumab is usually used sequentially with chemotherapy. Concurrent chemotherapy and trastuzumab could increase the treatment effect, which might explain the major discrepancies between settings. Besides, the method of HER2 signal counting might also influence the result. In the study from Perez et al, the maximum HER2 signal count for the form of clouds was assigned as 20 per cell. This approach would not change the decision for trastuzumab treatment, but could underestimate the amplification level of some patients.

The pharmacological mechanism of trastuzumab was reported mainly from two aspects. One was described as anti-HER2 dimerization and blockage of downstream cell signaling. The other was considered to be related to immune- and antibody-dependent cell-mediated cytotoxicity (ADCC). ${ }^{19}$ When trastuzumab binds to the FC $\gamma \mathrm{R}$ of natural killer cells, it would trigger ADCC and activate cell lysis. Some studies have reported that single-nucleotide polymorphisms of the $\mathrm{FC} \gamma \mathrm{R}$ might predict the efficacy of trastuzumab. ${ }^{20,21}$ One of the studies focused on the FC $\gamma \mathrm{R}$ gene FCGR3A and determined that single-nucleotide polymorphism rs396991 changed the amino acid on position 158 of FCGR3A. Patients with the low-affinity allele received less benefit from trastuzumab. Theoretically, a higher HER2 gene amplification level may lead to higher HER2 protein expression on the tumor cell surface. This is probably true considering the general consistency of the IHC and FISH results. At the same time, the tumor cell tends to combine with more antibodies, thus causing a greater extent of ADCC. Preclinical studies did determine that the ADCC effect of trastuzumab was partially relevant to HER2 expression levels on tumor target cells in BC patients. ${ }^{22}$ This was consistent with our result from the clinical samples.
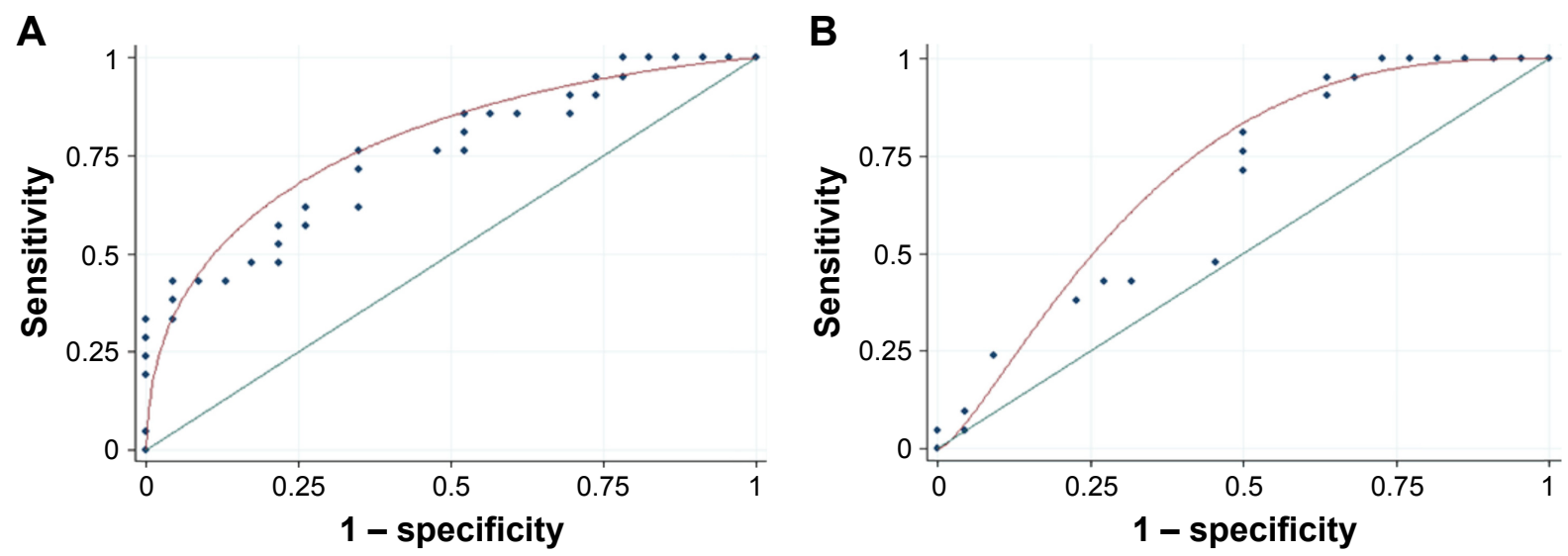

Figure 3 Optimal cutoff points of HER2/CEPI 7 ratio and HER2 GCN decided by ROC curves.

Notes: ROC curve using HER2/CEPI 7 ratio $(\mathbf{A})$ and HER2 GCN (B) to select the best responder to concurrent neoadjuvant chemotherapy and trastuzumab. The AUC for HER2/CEPI7 ratio was 0.786 , which was better than the AUC for HER2 GCN (0.656).

Abbreviations: GCN, gene copy number; ROC, receiver operating characteristic; AUC, area under the curve. 
Table 7 Summary of the relevant research

\begin{tabular}{|c|c|c|c|c|}
\hline Study & Settings & Treatment & Testing method & Conclusion \\
\hline Borley et al, ${ }^{5} 2014$ & Adjuvant & $\begin{array}{l}\text { Adjuvant therapy (details not } \\
\text { provided) }\end{array}$ & FISH (dual probe) & $\begin{array}{l}\text { High HER2 GCN associated with better } \\
\text { survival }\end{array}$ \\
\hline Xuan et al, ${ }^{4} 2015$ & & Trastuzumab-based chemotherapy & FISH (dual probe) & $\begin{array}{l}\text { Both GCN and HER2/CEPI7 ratio (RATIO) } \\
\text { associated with better survival }\end{array}$ \\
\hline Perez et al, ${ }^{3} 2014$ & & $\begin{array}{l}\text { Doxorubicin and } \\
\text { cyclophosphamide followed by } \\
\text { weekly paclitaxel with trastuzumab }\end{array}$ & FISH (dual probe) & $\begin{array}{l}\text { No correlations between survival and } \\
\text { RATIO/GCN }\end{array}$ \\
\hline Dowsett et al, ${ }^{6} 2009$ & & $\begin{array}{l}\text { Chemotherapy followed by } \\
\text { trastuzumab }\end{array}$ & FISH (dual probe) & $\begin{array}{l}\text { No correlations between survival and } \\
\text { RATIO/GCN }\end{array}$ \\
\hline Arnould et al," 2007 & Neoadjuvant & $\begin{array}{l}\text { Trastuzumab-based chemotherapy } \\
\text { with either docetaxel or docetaxel } \\
\text { plus carboplatin }\end{array}$ & $\begin{array}{l}\text { FISH (single } \\
\text { probe) }\end{array}$ & High GCN associated with $\mathrm{pCR}$ \\
\hline Buzdar et al, ${ }^{13} 2007$ & & $\begin{array}{l}\text { Trastuzumab-based chemotherapy } \\
\text { with paclitaxel followed by } \\
5 \text {-fluorouracil, epirubicin and } \\
\text { cyclophosphamide }\end{array}$ & FISH (dual probe) & $\begin{array}{l}\text { No correlations between } \mathrm{pCR} \text { and } \\
\text { RATIO/GCN }\end{array}$ \\
\hline Kogawa et al, ${ }^{18} 2016$ & & $\begin{array}{l}\text { Chemotherapy with an } \\
\text { anthracycline and/or a taxane } \\
\text { given in combination or sequential } \\
\text { regimens with or without } \\
\text { trastuzumab }\end{array}$ & FISH (dual probe) & High RATIO associated with $p C R$ \\
\hline Yu et al, ${ }^{12} 2012$ & & $\begin{array}{l}\text { Chemotherapy without } \\
\text { trastuzumab }\end{array}$ & FISH (dual probe) & High GCN associated with $\mathrm{pCR}$ \\
\hline Fuchs et al, ${ }^{8} 2014$ & Metastatic & Trastuzumab-based treatment & FISH (dual probe) & $\begin{array}{l}\text { High GCN associated with shorter time } \\
\text { to first metastasis }\end{array}$ \\
\hline Gullo et al, ${ }^{9} 2009$ & & Trastuzumab-based treatment & FISH (dual probe) & High RATIO associated with shorter TTP \\
\hline Kim et al, ${ }^{10} 2013$ & & $\begin{array}{l}\text { Trastuzumab plus taxane } \\
\text { chemotherapy }\end{array}$ & FISH (dual probe) & High RATIO associated with longer PFS \\
\hline
\end{tabular}

Abbreviations: FISH, fluorescence in situ hybridization; GCN, gene copy number; PCR, pathological complete response; TTP, time to progression; PFS, progressionfree survival.

Though the FISH result seemed simple and superficial, we should not neglect its value as a method for HER2 gene quantization and as a therapeutic predictor. A highlight of our study is that we could use this routinely conducted test as a predictive and prognosis marker in HER2-positive patients, if validated.

Trastuzumab and pertuzumab are dual-targeted drugs for HER2-positive BC in the neoadjuvant setting. However, pertuzumab is not approved in the People's Republic of China currently. Thus, only trastuzumab was used in all the patients. Another major limitation of our study is the relatively small sample size. However, we did observe an obvious trend between the HER2 amplification level and pCR results, despite the small sample size. Studies with larger sample sizes and assessing longer-term survival data are currently ongoing to validate our results in future studies.

\section{Conclusion}

In summary, our study demonstrated that while using the latest ASCO/CAP guideline for HER2 testing, a higher HER2 amplification level was correlated with larger tumor size and less ER and PR expression. In addition, both a higher HER2/ $C E P 17$ ratio and HER $2 \mathrm{GCN}$ could predict a better response to neoadjuvant therapy in trastuzumab-treated HER2-positive patients from neoadjuvant trials.

\section{Acknowledgment}

This work is supported by grants from the National Natural Science Foundation of the People's Republic of China (grant numbers 81172505 and 81302302), the Doctoral Programs Foundation of the Ministry of Education of People's Republic of China (grant number 20120071120105), the Shanghai Natural Science Foundation (grant number 13ZR1452800), the Shanghai Municipal Commission of Health and Family Planning (grant numbers 20144Y0218, 201640006), the Science and Technology Commission of Shanghai Municipality (grant number 14411950202), the Clinical Research Plan of SHDC (grant number SHDC 12016231), and the Nurturing Fund of Renji Hospital 2015 (grant number RJZZ15-023). The funders had no role in study design, data collection and analysis, decision to publish, or preparation of the manuscript. 


\section{Disclosure}

The authors report no conflicts of interest in this work.

\section{References}

1. Harris JR. HER2/ERBB2 Testing: Assessment of Status for Targeted Therapies. In: Harris JR, Lippman ME, Morrow M, Osborne CK, editors. Diseases of the Breast. 5th ed. Philadelphia: Lippincott Williams \& Wilkins; 2014:324-438.

2. National Comprehensive Cancer Network. NCCN Guidelines Version 2.2016: Breast Cancer.

3. Perez EA, Romond EH, Suman VJ, et al. Trastuzumab plus adjuvant chemotherapy for human epidermal growth factor receptor 2-positive breast cancer: planned joint analysis of overall survival from NSABP B-31 and NCCTG N9831. J Clin Oncol. 2014;32(33):3744-3752.

4. Xuan Q, Ji H, Tao X, et al. Quantitative assessment of HER2 amplification in HER2-positive breast cancer: its association with clinical outcomes. Breast Cancer Res Treat. 2015;150(3):581-588.

5. Borley A, Mercer T, Morgan M, et al. Impact of HER2 copy number in IHC2+/FISH-amplified breast cancer on outcome of adjuvant trastuzumab treatment in a large UK cancer network. Br J Cancer. 2014; 110(8):2139-2143.

6. Dowsett M, Procter M, McCaskill-Stevens W, et al. Disease-free survival according to degree of HER2 amplification for patients treated with adjuvant chemotherapy with or without 1 year of trastuzumab: the HERA Trial. J Clin Oncol. 2009;27(18):2962-2969.

7. Xu QQ, Pan B, Wang CJ, et al. HER2 amplification level is not a prognostic factor for HER2-positive breast cancer with trastuzumab-based adjuvant treatment: a systematic review and meta-analysis. Oncotarget. 2016;7(39):63571-63582.

8. Fuchs EM, Kostler WJ, Horvat R, et al. High-level ERBB2 gene amplification is associated with a particularly short time-to-metastasis, but results in a high rate of complete response once trastuzumab-based therapy is offered in the metastatic setting. Int J Cancer. 2014;135(1): 224-231.

9. Gullo G, Bettio D, Torri V, Masci G, Salvini P, Santoro A. Level of HER2/neu gene amplification as a predictive factor of response to trastuzumab-based therapy in patients with HER2-positive metastatic breast cancer. Invest New Drugs. 2009;27(2):179-183.

10. Kim JW, Kim JH, Im SA, et al. HER2/CEP17 ratio and HER2 immunohistochemistry predict clinical outcome after first-line trastuzumab plus taxane chemotherapy in patients with HER2 fluorescence in situ hybridization-positive metastatic breast cancer. Cancer Chemother Pharmacol. 2013;72(1):109-115.

11. Arnould L, Arveux P, Couturier J, et al. Pathologic complete response to trastuzumab-based neoadjuvant therapy is related to the level of HER-2 amplification. Clin Cancer Res. 2007;13(21):6404-6409.

12. Yu KD, Liu GY, Zhou XY, et al. Association of HER-2 copy number and HER-2/CEP-17 ratio with neoadjuvant taxane-containing chemotherapy sensitivity in locally advanced breast cancer. Oncologist. 2012;17(6): 792-800.
13. Buzdar AU, Valero V, Ibrahim NK, et al. Neoadjuvant therapy with paclitaxel followed by 5 -fluorouracil, epirubicin, and cyclophosphamide chemotherapy and concurrent trastuzumab in human epidermal growth factor receptor 2-positive operable breast cancer: an update of the initial randomized study population and data of additional patients treated with the same regimen. Clin Cancer Res. 2007;13(1):228-233.

14. Wolff AC, Hammond ME, Hicks DG, et al. Recommendations for human epidermal growth factor receptor 2 testing in breast cancer: American Society of Clinical Oncology/College of American Pathologists clinical practice guideline update. Arch Pathol Lab Med. 2014; 138(2):241-256.

15. Guiu S, Gauthier M, Coudert B, et al. Pathological complete response and survival according to the level of HER-2 amplification after trastuzumabbased neoadjuvant therapy for breast cancer. Br J Cancer. 2010; 103(9):1335-1342.

16. Wu K, Yang Q, Liu Y, Wu A, Yang Z. Meta-analysis on the association between pathologic complete response and triple-negative breast cancer after neoadjuvant chemotherapy. World J Surg Oncol. 2014;12:95.

17. Liedtke C, Mazouni C, Hess KR, et al. Response to neoadjuvant therapy and long-term survival in patients with triple-negative breast cancer. J Clin Oncol. 2008;26(8):1275-1281.

18. Kogawa T, Fouad TM, Liu DD, et al. High HER2/centromeric probe for chromosome 17 fluorescence in situ hybridization ratio predicts pathologic complete response and survival outcome in patients receiving neoadjuvant systemic therapy with trastuzumab for HER2-overexpressing locally advanced breast cancer. Oncologist. 2016;21(1):21-27.

19. Musolino A, Naldi N, Bortesi B, et al. Immunoglobulin G fragment $\mathrm{C}$ receptor polymorphisms and clinical efficacy of trastuzumab-based therapy in patients with HER-2/neu-positive metastatic breast cancer. $J$ Clin Oncol. 2008;26(11):1789-1796.

20. Gavin PG, Song N, Kim SR, et al. Association of polymorphisms in FCGR2A and FCGR3A with degree of trastuzumab benefit in the adjuvant treatment of ERBB2/HER2-positive breast cancer: analysis of the NSABP B-31 trial. JAMA Oncol. 2017;3(3):335-341.

21. Norton N, Olson RM, Pegram M, et al. Association studies of Fcgamma receptor polymorphisms with outcome in HER2+ breast cancer patients treated with trastuzumab in NCCTG (Alliance) trial N9831. Cancer Immunol Res. 2014;2(10):962-969.

22. Boero S, Morabito A, Banelli B, et al. Analysis of in vitro ADCC and clinical response to trastuzumab: possible relevance of FcgammaRIIIA/ FcgammaRIIA gene polymorphisms and HER-2 expression levels on breast cancer cell lines. $J$ Transl Med. 2015;13:324.

23. Wolff AC, Hammond ME, Schwartz JN, et al. American Society of Clinical Oncology/College of American Pathologists guideline recommendations for human epidermal growth factor receptor 2 testing in breast cancer. Arch Pathol Lab Med. 2007;131(1):18-43.
OncoTargets and Therapy

\section{Publish your work in this journal}

OncoTargets and Therapy is an international, peer-reviewed, open access journal focusing on the pathological basis of all cancers, potential targets for therapy and treatment protocols employed to improve the management of cancer patients. The journal also focuses on the impact of management programs and new therapeutic agents and protocols on

\section{Dovepress}

patient perspectives such as quality of life, adherence and satisfaction The manuscript management system is completely online and includes a very quick and fair peer-review system, which is all easy to use. Visit http://www.dovepress.com/testimonials.php to read real quotes from published authors. 